\title{
Theory of $Q$ control in atomic force microscopy
}

\author{
Tomás R. Rodríguez and Ricardo García ${ }^{a)}$ \\ Instituto de Microelectrónica de Madrid, CSIC, Isaac Newton 8, 28760 Tres Cantos, Madrid, Spain
}

(Received 13 February 2003; accepted 1 May 2003)

We discuss the performance of an atomic force microscope (AFM) operated in the amplitude modulation mode under a self-excitation signal, known as quality factor control ( $Q$ control). By using the point-mass description of the AFM, we provide a complete description of $Q$ control in tapping mode AFM. The theoretical simulations show three major results: (i) the steady-state motion of the system contains contributions from homogeneous and particular components, (ii) the active response of the microcantilever can be increased or decreased depending on the phase shift of the self-excitation with respect to the instantaneous deflexion, and (iii) in general, $Q$ enhancement reduces the maximum force exerted for the tip on the sample surface. (C) 2003 American Institute of Physics. [DOI: 10.1063/1.1584790]

Amplitude modulation (tapping mode) atomic force microscopy (AM-AFM) is characterized in many situations of interest by the coexistence of two oscillations states: a lowamplitude state usually dominated by attractive forces, and a high-amplitude state dominated by repulsive forces. ${ }^{1} \mathrm{Be}-$ cause in the high-amplitude state there is tip-surface mechanical contact, a major surface deformation can be expected. $^{2}$ To avoid this problem, Anczykowski and coworkers suggested that an effective increase of the quality factor $(Q)$ of the microcantilever could favor the oscillation in the noncontact state. ${ }^{3}$ To accomplish this the cantilever signal is amplified, phase shifted by $90^{\circ}$, and then fed back to the standard AM-AFM excitation force. An electronic modification of the effective $Q$ has also been applied to increase the speed of AM-AFM imaging. ${ }^{4}$ On the other hand, the hydrodynamic damping with the medium reduces the $Q$ by about two orders of magnitude, which gives values of 1-5 while operating in liquids. ${ }^{5}$ Low $Q$ values imply a slower response of the variations of the tip-to-surface forces, which in turn imply a global slower response of the system. To improve AM-AFM imaging in liquids, $Q$ control has also been applied. ${ }^{6-8}$ However, experimental results remain controversial concerning the usefulness of $Q$ enhancement. ${ }^{9}$

The controversy about $Q$ control can be attributed partly to the poor theoretical understanding of how it works. This can be traced back to the fact that all previous analyses have made two major and unproven hypotheses: (i) a steady-state solution with no contributions from the transients that led (ii) to the use of an approximate equation to describe AM-AFM under $Q$ control. In this letter, we solve the equation of a point-mass cantilever under an external excitation force, a tip-surface interaction, and a self-excitation gain without any approximation on the phase lag between self-excitation and the instantaneous deflexion of the microcantilever, with no hypothesis about the final solution. We demonstrate that a self-excitation with a phase lag of $90^{\circ}$ with respect to the instantaneous deflexion maximizes the $Q$ enhancement. We also demonstrate that estimations of the effective $Q$ based on approximative solutions tend to overestimate the $Q$ enhancement.

The general equation of a point-mass cantilever in AMAFM with a self-excitation gain and in absence of tipsurface forces is as follows:

$$
\ddot{z}(t)+\frac{\omega_{0}}{Q} \dot{z}(t)+\omega_{0}^{2} z(t)+\frac{k}{m} G z\left(t-\frac{\phi}{\omega}\right)=\frac{F_{0}}{m} \cos \omega t,
$$

where $z$ is the tip-cantilever's deflexion, $\omega_{0}$ is the angular free resonance frequency, $F_{0}$ is the driving force, $G$ is the gain factor of the self-excitation, $\phi$ is the phase shift between the self-excitation and the instantaneous deflexion, $m$ $=k / \omega_{0}^{2}$, and $k$ is the force constant of the cantilever. The general solution of Eq. (1) can be expressed by $z(t)=z_{h}(t)$ $+z_{p}(t)$, where $z_{h}(t)$ is the solution of the homogeneous equation and $z_{p}(t)$ is a particular solution of Eq. (1), which takes the form

$$
z_{p}(t)=A(\omega, G, \phi) \cos [\omega t-\varphi(\omega, G, \phi)]
$$

In previous works $3,4,6,7$ the contribution of $z_{h}(t)$ to the steady-state solution has been neglected. Furthermore, only a self-excitation of $\phi=90^{\circ}$ was considered, which makes the self-excitation term proportional to the cantilever's velocity and allows us to estimate the effective $Q$ from

$$
\frac{1}{Q_{\text {eff }}}=\frac{1}{Q}-\frac{k G}{m \omega \omega_{0}} .
$$

First, we perform a comparison between the numerical solution of the general Eq. (1) and the particular solution given by Eq. (2). To illustrate the active response of the microcantilever, we describe first the particular solution of Eq. (1). In this case, the amplitude can be expressed analytically:

$$
\begin{gathered}
A(\omega, G, \phi) \\
\sqrt{\left(\omega_{0}^{2}-\omega^{2}+\frac{k G \cos \phi}{m}\right)^{2}+\left(\frac{\omega \omega_{0}}{Q}-\frac{k G \sin \phi}{m}\right)^{2}}
\end{gathered}
$$

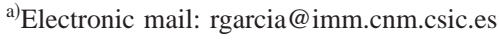



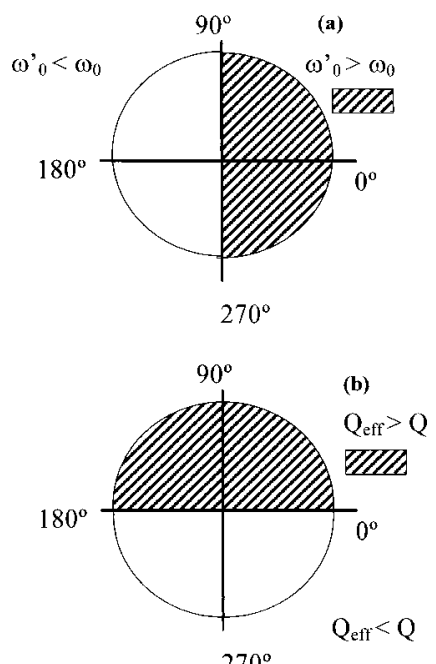

FIG. 1. Map of the qualitative changes of the resonance frequency (a) and $Q$ (b) of the self-excited microcantilever system.

This expression shows that in addition to the dependence on $\omega, \omega_{0}$, and $Q$, the amplitude also depends on $G$ and $\phi$. By comparison with the Lorenztian of a forced oscillator with damping, it can be deduced that the self-excitation modifies both the resonance frequency and the effective $Q$. Phase shifts between $0^{\circ}$ and $90^{\circ}$ and $270^{\circ}$ and $360^{\circ}$ displace the new resonance frequency to higher values with respect to resonance frequency of the non-self-excited system $\left(\omega_{0}^{\prime}\right.$ $\left.>\omega_{0}\right)$ while other phase shifts decrease the resonance frequency [Fig. 1(a)]. At $\phi=90^{\circ}$ and $270^{\circ}$, there are no modifications of the resonance frequency, $\omega_{0}^{\prime}=\omega_{0}$. On the other hand, $Q_{\text {eff }}$ is increased by applying a phase shift between $0^{\circ}$ and $180^{\circ}$ [Fig. 1(b)].

Equation (4) shows that $\phi=90^{\circ}$ is the optimum phase shift for the self-excitation because it does not modify the resonance frequency of the non-self-excited system, and at the same time it maximizes the effective $Q$. The tendency described in Fig. 1 is reproduced by the general solution $z(t)=z_{h}+z_{p}$. In what follows, the calculations are performed for $\phi=90^{\circ}$.

The effect of $G$ on the amplitude curves at $\phi=90^{\circ}$ and at constant driving force is shown in Fig. 2. The curve of the non-self-excited system, with a maximum of about $10 \mathrm{~nm}$, appears as a straight line at the scale of the figure. Increasing $G$ increases the oscillation amplitude, shifts the resonance

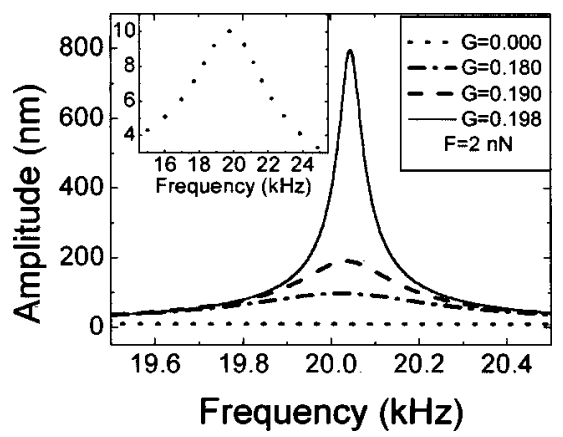

FIG. 2. Amplitude of the general solution vs frequency for different values of the self-excitation gain. The curves have been obtained for a $F_{0}=2 \mathrm{nN}$, $k=1 \mathrm{~N} / \mathrm{m}, \omega_{0} / 2 \pi=20 \mathrm{kHz}$, and $Q=5$. The inset shows the resonance curve of the non-self-excited system.

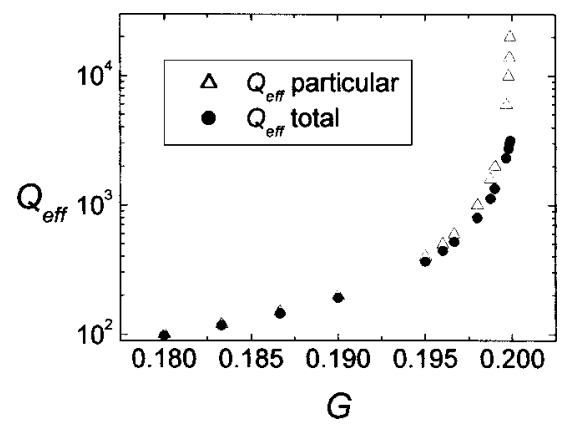

FIG. 3. Dependence of $Q_{\text {eff }}$ as a function of $G$. Solid and open symbols correspond to the general and particular solutions of Eq. (1) respectively; $\phi=90^{\circ}$.

frequency to higher values, and narrows the amplitude curves. The amplitude at resonance can be increased by two orders of magnitude with respect to the initial system, while the resonance frequency changes by a small factor $(0.3 \%)$. An increase of $G$ from 0 to 0.198 produces an increase of $Q_{\text {eff }}$ of about two orders of magnitude (from 5 to 403). These results were obtained by numerically solving Eq. (1) with a fourth Runge-Kutta algorithm. Equation (1) was integrated over a large number of periods $(\sim 5000)$ until a steady-state solution was reached. $Q_{\text {eff }}$ was determined by adjusting the resonance curve to the Lorenztian form of a forced oscillator with damping. ${ }^{10}$

The effect of gain in the $Q$ is sensitive to the solutions of Eq. (1) considered, either general or particular. In Fig. 3, the effective $Q$ is plotted as a function of $G$ for the $z(t)=z_{h}$ $+z_{p}$ and $z_{p}(t)$ solutions, respectively. At relatively low $G$, there are hardly any differences. However, for higher $G$, the particular solution overestimates by a factor close to 10 the enhancement of the $Q$. The above behavior is unequivocally related to the presence of a non-negligible homogeneous term in the general solution and questions the numerical relevance of previous analyses. ${ }^{3,6,7}$ Those effects are even more pronounced when the self-excitation is approximated by a term proportional to the velocity: $G z(t-\pi / 2 \omega)=$ $-\omega^{-1} G[\dot{z}(t)]$. Then,

$$
\ddot{z}(t)+\frac{\omega_{0}}{Q} \dot{z}(t)+\omega_{0}^{2} z(t)-\frac{k}{\omega m} G \dot{z}(t)=\frac{F_{0}}{m} \cos \omega t .
$$

The general solution of this equation is

$$
\begin{aligned}
z(t)= & z_{h}(t)+z_{p}(t)=e^{-\beta t}(C \cos \varpi t+D \sin \varpi t) \\
& +A(\omega) \cos [\omega t-\varphi(\omega)],
\end{aligned}
$$

with

$$
\beta=\frac{\omega_{0}}{2 Q}-\frac{k G}{2 m \omega}
$$

and

$$
\varpi=\omega_{0} \sqrt{1-\frac{1}{4}\left(\frac{1}{Q}-\frac{k G}{m \omega_{0} \omega}\right)^{2}} .
$$

Equation (8) shows an unstable behavior whenever

$$
\frac{\omega}{\omega_{0}}<\left(1-\frac{Q}{Q_{\text {eff }}}\right)
$$


for example, for moderate $Q$ enhancements, such as $Q_{\text {eff }}$ $=10 Q$, the system will diverge for $\omega=0.9 \omega_{0}$. All of these equations emphasize the relevance of homogeneous contribution to describe the dynamic response of the system.

To study the effect of the self-excitation in the presence of tip-surface forces, we have modeled the AFM interface following the approximations given in Ref. 11. The result of the simulations show that the slope of amplitude curve decreases with decreasing the tip-surface separation. However, the slope of the amplitude curve is substantially larger for the self-excited system which, in principle, implies a higher sensitivity to tip-surface variations. The measurement of the maximum force offers a good argument in favor of the use of $Q$ enhancement because, for identical tip-surface separations, the self-excited system experiences a reduction of the force by a factor of 5 to 6 . The same result is obtained if the average force is calculated instead. The simulations are in agreement with the expression of the average force as a function of the set point $\left(A_{\mathrm{sp}}\right)$ and free amplitude $\left(A_{0}\right)$, that can be deduced by applying energy considerations and the virial theorem to the tip motion: $:^{1,12}$

$$
\left\langle F_{\mathrm{ts}}\right\rangle=\frac{k}{Q_{\mathrm{eff}}}\left[A_{0}^{2}-A_{\mathrm{sp}}^{2}\right]^{1 / 2} .
$$

In short, we have presented a complete description of the active response of the microcantilever in amplitude modulation AFM under a self-excitation force. Our description shows that the homogeneous component of the solution does influence the steady-state oscillation and as a consequence, its omission leads to inaccurate or wrong quantitative results. The self-excitation force may speed up or slow down the dynamic response of the system depending on the phase shift between the self-excitation and the instantaneous deflexion. Finally, we have demonstrated that an enhanced quality factor minimizes tip-surface forces during tapping mode operation.

This work was financially supported by the European Commission (MONA-LISA, GR5RD-2000-00349) and the Dirección General de Investigación Científica y Técnica (Spain) (PB98-0471).

${ }^{1}$ R. García and R. Pérez, Surf. Sci. Rep. 47, 197 (2002).

${ }^{2}$ A. San Paulo and R. García, Biophys. J. 78, 1599 (2000).

${ }^{3}$ B. Anczykowski, J. P. Cleveland, D. Krüger, V. Elings, and H. Fuchs, Appl. Phys. A: Mater. Sci. Process. 66, S885 (1998).

${ }^{4}$ T. Sulchek, R. Hsieh, J. D. Adams, G. G. Yaraioglu, S. C. Minne, C. F. Quate, J. P. Cleveland, A. Atalar, and D. M. Adderton, Appl. Phys. Lett. 76, 1473 (2000).

${ }^{5}$ T. E. Schäffer, J. P. Cleveland, F. Ohnesorge, D. A. Walters, and P. K. Hansma, J. Appl. Phys. 80, 3622 (1996).

${ }^{6}$ J. Tamayo, A. D. L. Humphris, and M. J. Miles, Appl. Phys. Lett. 77, 582 (2000).

${ }^{7}$ J. Tamayo, A. D. L. Humpris, R. J. Owen, and M. J. Miles, Biophys. J. 81, 526 (2001).

${ }^{8}$ T. R. Rodríguez and R. García, Patent No. 200201 022, Spanish Patent Office.

${ }^{9}$ R. D. Jäggi, A. Franco-Obregón, P. Studerus, and K. Ensslin, Appl. Phys. Lett. 79, 135 (2001).

${ }^{10}$ A. P. French, Vibrations and Waves, The MIT Introductory Physics Series (Norton, New York, 1971).

${ }^{11}$ R. García and A. San Paulo, Phys. Rev. B 60, 4961 (1999).

${ }^{12}$ A. San Paulo and R. García, Phys. Rev. B 64, 193411 (2001). 